\title{
Static Magnetic Field Stimulation over the Visual Cortex Increases Alpha Oscillations and Slows Visual Search in Humans
}

\author{
Javier J. Gonzalez-Rosa, ${ }^{1}$ Vanesa Soto-Leon, ${ }^{2}$ Pablo Real, ${ }^{1}$ Carmen Carrasco-Lopez, ${ }^{2}$ Guglielmo Foffani, ${ }^{3,4}$ \\ Bryan A. Strange, ${ }^{1,5}$ and Antonio Oliviero ${ }^{2}$ \\ ${ }^{1}$ Laboratory for Clinical Neuroscience, Centre of Biomedical Technology, Technical University of Madrid, 28223 Madrid, Spain, ${ }^{2}$ FENNSI Group, and \\ ${ }^{3}$ Neural Bioengineering Group, Hospital Nacional de Parapléjicos, SESCAM, 45071 Toledo, Spain, ${ }^{4}$ Centro Integral de Neurociencias A.C., HM Puerta del \\ Sur, Hospitales de Madrid, Móstoles and Medical School, CEU-San Pablo University, 28938 Madrid, Spain, and ${ }^{5}$ Department of Neuroimaging, Reina Sofia \\ Centre for Alzheimer's Research, 28031 Madrid, Spain
}

Transcranial static magnetic field stimulation (tSMS) was recently introduced as a promising tool to modulate human cerebral excitability in a noninvasive and portable way. However, a demonstration that static magnetic fields can influence human brain activity and behavior is currently lacking, despite evidence that static magnetic fields interfere with neuronal function in animals. Here we show that transcranial application of a static magnetic field $(120-200 \mathrm{mT}$ at 2-3 cm from the magnet surface) over the human occiput produces a focal increase in the power of alpha oscillations in underlying cortex. Critically, this neurophysiological effect of tSMS is paralleled by slowed performance in a visual search task, selectively for the most difficult target detection trials. The typical relationship between prestimulus alpha power over posterior cortical areas and reaction time (RT) to targets during tSMS is altered such that tSMS-dependent increases in alpha power are associated with longer RTs for difficult, but not easy, target detection trials. Our results directly demonstrate that a powerful magnet placed on the scalp modulates normal brain activity and induces behavioral changes in humans.

Key words: EEG; NIBS; occipital cortex; static magnetic field; TMS; visual search

\section{Introduction}

Magnets have been used in attempts to treat disorders of the nervous system since Aetius of Amida (Häfeli, 2007). However, modern neuroscience has seen limited exploration of the effects of static magnetic fields on human brain function (Roberts et al., 2011), despite evidence that static magnetic fields interfere with neural function in animals (Rosen and Lubowsky, 1987, 1990; McLean et al., 2003, 2008; Yang et al., 2011; Wu and Dickman, 2012; Aguila et al., 2014). We recently demonstrated that applying transcranial static magnetic field stimulation (tSMS) in humans reduces the excitability of the motor cortex, as measured by a reduced amplitude of motor-evoked potentials elicited by transcranial magnetic stimulation (TMS; Oliviero et al., 2011)—a result that has

Received Oct. 12, 2014; revised May 3, 2015; accepted May 8, 2015.

Author contributions: J.J.G.-R., G.F., B.A.S., and A.O. designed research; J.J.G.-R., V.S.-L., P.R., and C.C.-L. performed research; J.J.G.-R., V.S.-L., G.F., and A.O. analyzed data; J.J.G.-R., G.F., B.A.S., and A.O. wrote the paper.

This research was funded by the "Ministerio de Economía y Competitividad" of Spain and the "Fondo Europeo de Desarrollo Regional-FEDER" (project SAF2012-39589). J.J.G.-R. is supported by a Marie Curie fellowship (FP7COFUND program). B.A.S. is supported by a Marie Curie Career Integration Grant (FP7-PEOPLE-2011-CIG 304248) and Grant SAF2011-27766 from the Spanish Ministry of Science and Innovation.

G.F. and A.O. declare that they are cofounders of the company Neurek SL, which is a spinoff of the Foundation of the Hospital Nacional de Parapléjicos. Moreover, they are inventors listed on the following patents: P201030610 and PCT/ES2011/070290 (patent abandoned). The authors declare no other competing financial interests.

Correspondence should be addressed to Antonio Oliviero, Hospital Nacional de Parapléjicos, FENNSI Group, Finca La Peraleda s/n, 45071 Toledo, Spain. E-mail: antonioo@sescam.jccm.es or antonio.oliviero@hotmail.com.

DOI:10.1523/JNEUROSCI.4232-14.2015

Copyright $\odot 2015$ the authors $\quad 0270-6474 / 15 / 359182-12 \$ 15.00 / 0$ been subsequently replicated (Silbert et al., 2013). However, direct measurements of the impact of tSMS on human brain activity and behavior are presently lacking.

Considering that tSMS alters normal functioning of membrane ion channels (Wieraszko, 2000; Rosen, 2003, 2010; Yang et al., 2011) and that the latter are critical for neuronal oscillations (Wang, 2010), we hypothesized that tSMS is able to modulate electroencephalogram (EEG) activity. We were specifically interested in the EEG oscillations in the alpha frequency range (7-14 $\mathrm{Hz}$ ), which is usually most pronounced bilaterally in posterior regions of the head and suppressed by eye opening (Niedermeyer, 1993; Barry et al., 2007). Alpha-band EEG activity has been shown to represent the state of visual cortex excitability (Pfurtscheller, 2001, 2003), as measured by phosphenes induced by TMS, with stronger alpha power corresponding to reduced cortical excitability (Romei et al., 2008a,b). We thus hypothesized that a reduction of cortical excitability induced by tSMS of the visual cortex would be expressed as an increase of alpha power.

We investigated the electrophysiological and behavioral effects of tSMS over the occipital cortex. We specifically tested whether a tSMS-dependent decrease in cortical excitability is reflected in a modulation of posterior alpha oscillations (Fig. 1A). Posterior alpha oscillations play an important role in visual perception (VanRullen and Macdonald, 2012) and selective visual attention (Foxe et al., 1998). In particular, ongoing alpha oscillations influence successful detection of a predefined target in vi- 

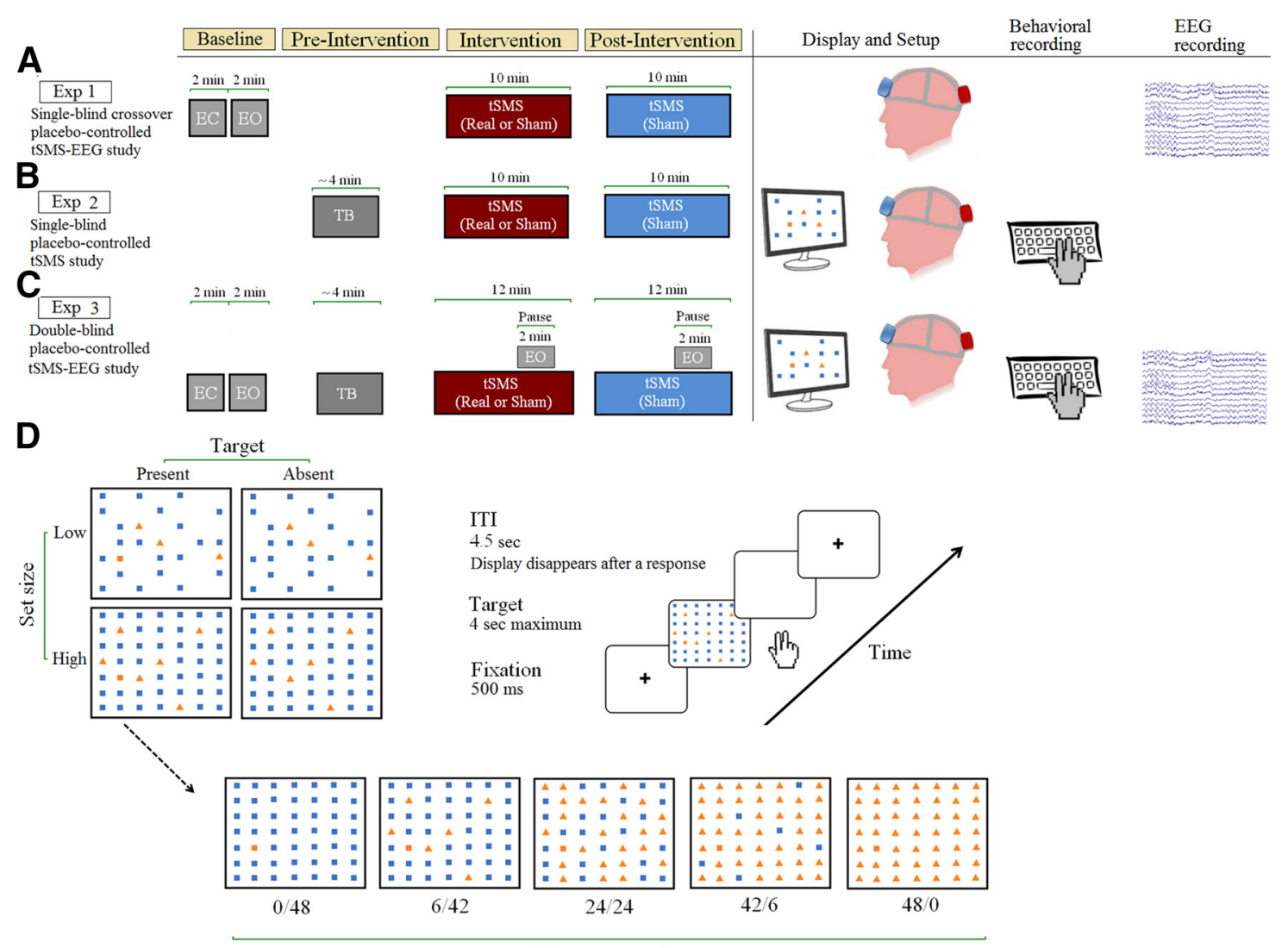

Distractor ratios

Figure 1. Experimental protocols. $\boldsymbol{A}-\boldsymbol{C}$, Time line (left) and experimental setup (right) for Experiments 1-3. $\boldsymbol{A}$, In Experiment 1, resting EEG alpha activity was measured throughout the experiment. $\boldsymbol{B}$, In Experiment 2, only behavioral data were collected during the performance of the conjunction visual search task. $\boldsymbol{C}$, In Experiment 3 , EEG alpha activity was measured during task performance, in addition to resting $\mathrm{EEG}$ measures during baseline and during $2 \mathrm{~min}$ "pause" time windows during intervention and postintervention sessions. EC, Eyes closed; $\mathrm{E} 0$, eyes open; $\mathrm{TB}$, task baseline. Red cylinder shows the scalp position over which the magnetic NdFeB cylinder (real-tSMS) or the nonmagnetic steel cylinder (sham-tSMS) was located over the visual cortex. Blue cylinder shows the scalp position over which a nonmagnetic steel cylinder was always located over the frontal cortex (around Fpz location) as a counterweight to the occipital cylinder. D, Top left, Example of a representative distractor ratio in stimulus array and all possible conditions for each distractor ratio (set size, and target presence/absence). Top right, Schematic of time course of an example trial (high set size, 6:42 distractor ratio, and target present). Bottom, Example of target-present trials in the 0:48, 6:42, 48:0, 24:24, and 42:6 distractor ratio conditions. ITI, Intertrial interval; Exp, experiment.

sual selective attention tasks (Dugué et al., 2011; Foxe and Snyder, 2011), with higher prestimulus alpha power associated with less likely, or slower, visual target detection (Thut et al., 2006; Hanslmayr et al., 2007). Thus, we also tested whether tSMS of the visual cortex modulates visual search performance in a conjunction search task with a distractor-ratio manipulation (Fig. $1 B, D$; Wolfe et al., 1989; Poisson and Wilkinson, 1992). Finally, we repeated the visual search task while simultaneously recording EEG (Fig. 1C) to examine the relationship between tSMSdependent behavioral changes and tSMS-dependent increase in prestimulus alpha power.

\section{Materials and Methods}

A total of 76 right-handed subjects (39 women) with normal or corrected-to-normal visual acuity, no history of neurological or psychiatric disorders, and without any exclusion criteria for tSMS and TMS participated in our study. In Experiment 1, participants completed a single-blind, crossover, placebo-controlled tSMS-EEG study. In Experiment 2, participants were randomized to two equal-sized groups (real and sham, single-blind placebo-controlled tSMS study). In Experiment 3 , a new group of participants completed a double-blind placebocontrolled tSMS-EEG study. In Experiment 2 and Experiment 3, a between-subjects design was used to avoid potential learning effects across repeated sessions. There are no differences across groups regarding sex, age, or educational level. Participants were recruited by adver- tisement and gave written informed consent to participate in the study, which was approved by the local ethics committee of the "Hospital Nacional de Parapléjicos" of Toledo (Spain).

\section{Transcranial static magnetic stimulation}

The magnet used for tSMS in all experiments was a cylindrical nickelplated (Ni-Cu-Ni) NdFeB magnet of $60 \mathrm{~mm}$ diameter, $30 \mathrm{~mm}$ thickness, and a weight of $670 \mathrm{~g}$ (model Mag60r; custom-made by Supermagnete for Neurek SL). The nominal strength of this magnet is $120 \mathrm{~kg}$, with an intensity of the magnetic field at the surface of the magnet, on the cylinder axis, of $0.45 \mathrm{~T}$ (Rivadulla et al., 2014). At $2-3 \mathrm{~cm}$ from the magnet surface (i.e., the approximate depth of the visual cortex from the scalp) the magnetic field strength of this magnet on the cylinder axis is 120-200 $\mathrm{mT}$ (Rivadulla et al., 2014). Because our previous work (Oliviero et al., 2011) showed that tSMS-dependent reduction of cortical excitability did not depend on polarity, the magnetic field polarity was set randomly north or south unless otherwise specified. A nonmagnetic metal cylinder, of the same size, weight, and appearance as the real magnet (Mag60s, Neurek SL), was used for sham stimulation. An additional nonmagnetic metal cylinder was always placed on the frontal cortex as a counterweight. We recently showed that $\mathrm{tSMS}$ is a safe procedure in humans (Oliviero et al., 2014).

\section{Experiment 1}

Subjects and procedure. The experimental procedure is depicted in Figure $1 A$ (Experiment 1). Sixteen participants (6 women; age range, $24-45$ 
years; mean age, 32.9 years; SD $=6.3$ ) completed a single-blind, crossover, placebo-controlled tSMS-EEG study. Each subject underwent both sham and real stimulation on separate days (order counterbalanced across subjects), but at least 1 week apart, at the same hour of the day. Participants were seated comfortably in a dimly lit, sound-attenuated, and electrically shielded room. A video camera was used to record the experiment and monitor the eye movements of the participants, who were asked to relax quietly while the spontaneous EEG activity at rest was recorded for the different experimental conditions: (1) during $4 \mathrm{~min}$ baseline ( 2 min eyes closed and 2 min eyes open; "baseline"); (2) during a tSMS (real or sham) session for 10 min ("intervention"); and (3) for 10 additional minutes after removing the magnet ("postintervention"). A nonmagnetic metal cylinder was placed over the visual cortex at the beginning of the baseline and was replaced by the real magnet or the sham magnet (i.e., another nonmagnetic metal cylinder) for the intervention, and the original nonmagnetic metal cylinder was placed again at the end of the intervention. The EEG session lasted $24 \mathrm{~min}$ in total.

Data acquisition. Simultaneous EEG activity was recorded using a 21channel EEG cap with built-in $\mathrm{Ag} / \mathrm{AgCl}$ electrodes (Electro-Cap International, Inc.). Electrode positions were located according to the 10/20 EEG International System (Fp1, Fpz, Fp2, F7, F3, Fz, F4, F8, T3, C3, Cz, C4, T4, T5, P3, Pz, T4, T6, POz, O1, and $\mathrm{O}_{2}$ ), plus two electrocardiogram (ECG) electrodes (one ECG electrode was placed on the sternum and the other over the precordium). Impedance was kept below $8 \mathrm{k} \Omega$. EEG signals were recorded using a montage with earlobe electrodes serving as references, and by digital EEG equipment (SAM 32, MicroMed; bandpass filter, 0.1-128 Hz; sampling frequency, $256 \mathrm{~Hz}$ ).

Data analysis. Data analysis was performed using MATLAB (MathWorks) and EEGLAB (http://sccn.ucsd.edu/eeglab). EEG signals were visually inspected for artifacts, and epochs containing large artifacts such as large blinks or gross motor movements were discarded from further analysis. To remove smaller eye-blink artifacts, an independent component analysis procedure was used (Makeig et al., 1996). Power spectral density was estimated using Welch's averaged modified periodogram method (linear detrending, no overlapping, Hamming windowing, 0.5 $\mathrm{Hz}$ frequency resolution). For each condition, single-segment power spectra were computed via FFT and then averaged to yield the mean power spectrum. Individual alpha frequency (IAF) was estimated from the mean spectrum over posterior scalp sites by means of peak detection between 7 and $14 \mathrm{~Hz}$. The mean spectral amplitude within the frequency range of the IAF $\pm 2 \mathrm{~Hz}$ (Klimesch, 1999) was calculated and log transformed. Spectral estimates of relative alpha power during intervention and postintervention at an electrode $i$ were then obtained for each subject by subtracting the log-transformed alpha power during open-eyes baseline $\left(\right.$ Pow $\left._{i, \text { baseline }}\right)$ from the log-transformed alpha power during intervention $\left(\right.$ Pow $_{i, \text { intervention }}$ ), or postintervention, according to the following formula:

$$
\text { Relative Alpha Power }(i)=\log \left(\operatorname{Pow}_{i, \text { intervention }}\right)-\log \left(\operatorname{Pow}_{i, \text { baseline }}\right) \text {. }
$$

Therefore, increases in power from baseline during and after the intervention are expressed as positive values. The resulting relative alpha power was collapsed across successive $150 \mathrm{~s}$ time bins for analysis purposes [yielding four arbitrary time bins during intervention (T1, T2, T3, $\mathrm{T} 4)$ and postintervention (T5, T6, T7, T8)].

Experimental debrief revealed that participants were not able to recognize whether the metallic cylinder used during intervention was the real magnet or sham (46.9\% of correct guesses; Pearson $\chi^{2}$ test, $p=0.71)$.

\section{Experiment 2}

Subjects and procedure. Thirty participants (17 women; age range, 23-42 years; mean age, 29.3 years; SD, 4.7) were randomized to two equal-sized groups (real and sham single-blind, placebo-controlled tSMS study; Fig. $1 B)$. Each subject, therefore, underwent either $\operatorname{sham}(n=15)$ or real $(n=$ 15) stimulation. One participant was excluded from the analyses due to technical problems during the data acquisition. To keep the distance from magnet to scalp equivalent to that in Experiment 1, participants in Experiment 2 wore the same EEG electrode cap during behavioral testing (without any EEG recordings) as in Experiment 1.
Conjunction visual search task. Three different stimuli were used to create the visual search displays: a light blue square, an orange triangle, and an orange square (Fig. 1D). Stimuli of different colors were matched in luminance and presented on a white background. The target was always an orange square (i.e., a specific combination of color and shape). Therefore, the orange triangle served as a same-color distractor while the light blue square served as a same-shape distractor. At a viewing distance of $60 \mathrm{~cm}$, each individual stimulus subtended a visual angle of $0.57^{\circ}(20 \times$ 13 pixels) horizontally and vertically. All stimuli were presented in a $17.52^{\circ} \times 12.36^{\circ}$ field $(621 \times 271$ pixels $)$, and the minimum distance between the centers of neighboring items was $2.86^{\circ}$ horizontally and $2^{\circ}$ vertically.

In visual search tasks, an observer searches for a predefined target in an array containing distractor objects, and the primary dependent variable is the function relating reaction time (RT) to the number of items in the display ("set size"; Wolfe et al., 1989) or to the relative frequency of the two types or subsets of distractors ("distractor ratio effect"; Wolfe et al., 1989; Treisman and Sato, 1990; Poisson and Wilkinson, 1992; Shen et al., 2003). Thus, the experiment was a distractor ratio and set size by "target presence" factorial design. That is, the first factor was the number of objects (set size) according to the fixed total number of stimuli that were presented in the display. In all of the conditions, the total number of stimuli was always fixed at 24 ("low set size") or 48 ("high set size"). The second factor was the presence or absence of target according to whether the target appeared in the display or not. For each trial, the relative frequency of the two types of distractors (same-shape/same-color) was manipulated across the following five possible distractor ratios: 0:24, $3: 21,12: 12,21: 3$, and 24:0 for trials with low set size, and 0:48, 6:42, 24:24, 42:6, and 48:0 for trials with high set size. For simplicity, in the text we only use high set size values when we refer to set distractor. The display was divided into a $7 \times 7$ imaginary grid box of 49 invisible regions. For target-present trials, once the display was generated, one of the distractors was randomly chosen to be replaced by the target stimulus (orange square). Therefore, in each display the total maximum number of distractors was 48 (49 for target-absent trials). Low- and high-set size conditions occurred with equal probability (50\%), whereas target-present or target-absent conditions occurred with a 3:1 probability (75\% and 25\%, respectively). For target-present trials, the target could appear at any location among all possible arrays of items. The specific combination was assigned at random to each participant, and trial-type order and position of items within stimulus arrays was pseudorandomized with the constraint that all possible combinations of set size, distractor ratio, and target presence had to occur every 40 trials $(\sim 4 \mathrm{~min})$. This means that for every intervention session we had three consecutive blocks of $\sim 3-4$ min each for a total of $\sim 10 \mathrm{~min}$ of recording.

The task began with a $\sim 4$ min preintervention session, followed by a 10 min intervention session (tSMS or sham), and then by a $10 \mathrm{~min}$ postintervention session. Each trial started with a black fixation cross presented centrally for $500 \mathrm{~ms}$, followed by the search display, which remained on screen until the subject responded or until a maximum of $4 \mathrm{~s}$ had elapsed. The maximum duration of each trial was therefore $4.5 \mathrm{~s}$, and there were a total of 120 trials during each session (intervention and postintervention). Participants were instructed to maintain central eye fixation when the fixation cross stayed on the screen, at the beginning of each new trial. After a response was given, the stimulus display was replaced by a blank screen until the next trial. To reduce the possibility of a speed-accuracy trade-off, participants were asked to locate as quickly as possible the target stimulus via forced choice (i.e., they pressed one of two keys to indicate target presence or absence, respectively). Behavioral performance was assessed by reaction time and the number of errors. The experiment lasted $24 \mathrm{~min}$ in total.

A postexperimental debriefing confirmed that participants were not able to detect whether the metallic cylinder used during the intervention was the real magnet or the sham (58.6\% of correct guesses; Pearson $\chi^{2}$ test, $p=0.36$ )

\section{Experiment 3}

Subjects and procedure. Thirty participants (16 women; age range, 18-31 years; mean age, 24.1 years; SD, 3.1) completed a double-blind, placebo- 
A

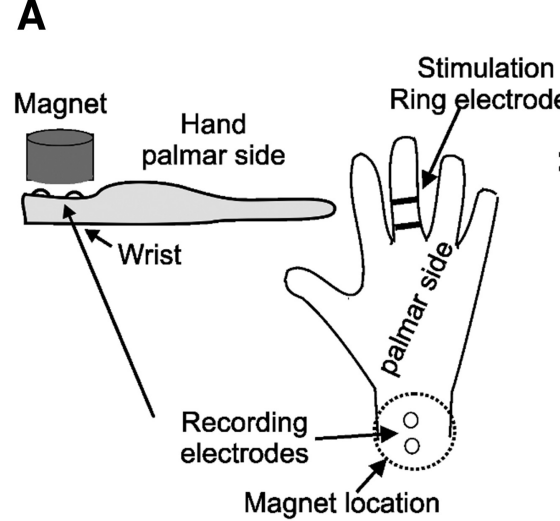

B

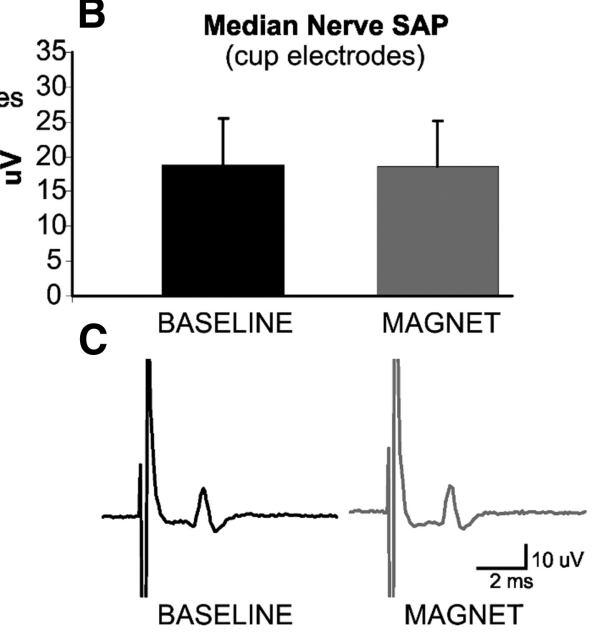

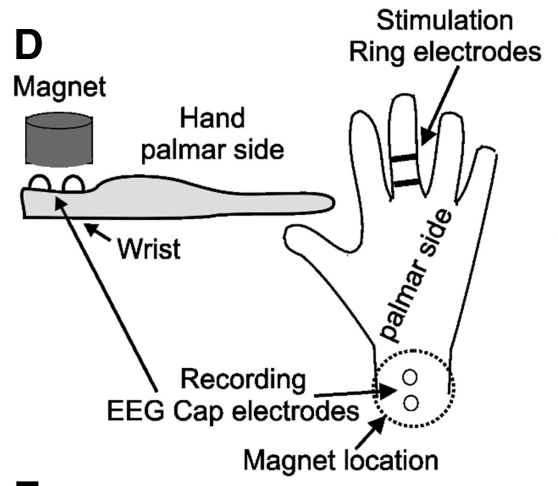

$\mathbf{E}$

Stimulation protocol

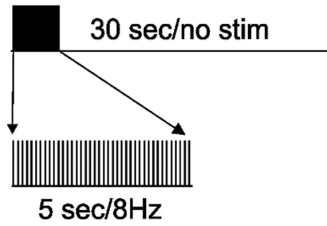

$\mathbf{F}$

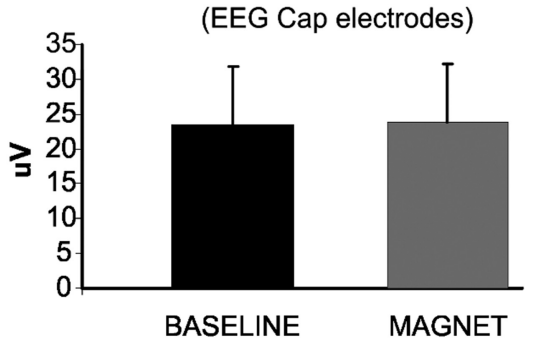

G

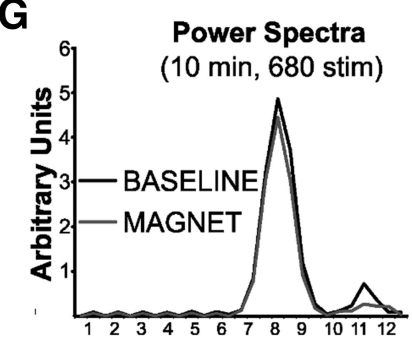

Figure 2. Control experiments. $A$, Schematic representation of the experimental setup of Experiment 4.1. $B$, SAP amplitudes, using standard cup electrodes, were identical during baseline and SMS. C, SAPs from a representative subject. $\boldsymbol{D}$, Schematic representation of the experimental setup of Experiment 4.2. $\boldsymbol{E}$, The stimulation protocol was a train of $40 \mathrm{stimuli}$ at $8 \mathrm{~Hz}(5 \mathrm{~s})$ followed by 30 s of rest. This protocol was repeated 17 times in each condition (baseline and SMS).F, SAP amplitudes, using EEG cap electrodes, were identical in baseline and during SMS. G, Power spectra showing the artificially induced $8 \mathrm{~Hz}$ SAP oscillations; these were identical during baseline and SMS.

controlled EEG-tSMS study (Fig. 1C). Each subject underwent either sham $(n=15)$ or real $(n=15)$ stimulation. Experiment 3 was designed to replicate and integrate the results of Experiments 1 and 2 by simultaneous recording of brain EEG activity and behavioral performance during tSMS stimulation. The behavioral setup of Experiment 2 was thus replicated by using the same task paradigm, adding simultaneous EEG recordings.

The most critical modification with regard to Experiments 1 and 2 was, as stated above, that the protocol was double blind instead of single blind. Additionally, the magnetic field polarity during tSMS was held constant (always south pointing toward the visual cortex), and an opaque plastic film was also interposed between the magnet (real or sham) and the EEG cap to avoid any contact between the gel and the metal cylinder. To obtain a measure of resting EEG alpha power during the intervention, a 2 min pause period was inserted after two-thirds of the task had been completed (for intervention and postintervention periods). This resulted in participants receiving a tSMS (real or sham) session for $\sim 12 \mathrm{~min}$. Because tSMS was shown to reduce motor cortex excitability both with 10 min durations (Oliviero et al., 2011) and 15 min durations (Silbert et al., 2013), we considered the difference between $10 \mathrm{~min}$ (Experiments 1 and 2) and 12 min (Experiment 3 ) negligible for the final results of the present study. In addition to recording $4 \mathrm{~min}$ baseline EEG activity ( 2 min with eyes closed and $2 \mathrm{~min}$ with eyes open) and before tSMS (as done in Experiment 1), EEG activity was also recorded during $\sim 4$ min of preintervention task performance. Thus, Experiment 3 lasted $32 \mathrm{~min}$ in total.

Data analysis. EEG data analysis was performed as in Experiment 1, but for each subject alpha power was normalized to the preintervention period for task periods and to the eyesopen baseline for pause periods. Furthermore, single-trial prestimulus oscillatory activity in the alpha band was also assessed to investigate the relationship between tSMS-dependent changes in alpha activity and tSMS-dependent changes in behavioral performance. Based on previous studies (Foxe et al., 1998; Thut et al., 2006; Hanslmayr et al., 2007), we focused on the time window of $1000-500 \mathrm{~ms}$ before target onset, during which alpha band power changes have been shown to predict visual perception performance (Hanslmayr et al., 2007; van Dijk et al., 2008; Foxe and Snyder, 2011). Consistent with previous evidence showing strong connections between ongoing alpha oscillations and the generation of event-related brain potentials (Basar, 1980; Klimesch et al., 2007b; Min et al., 2007), we did not analyze the last interval of pretarget activity ( $500 \mathrm{~ms}$ before target stimulus onset) to avoid any influence of fixation cross presentation on alpha activity, which could add variability to the data. Single-trial prestimulus alpha power was obtained by $8-14 \mathrm{~Hz}$ bandpass filtering. For each subject, the trials of the visual search task were sorted according to prestimulus alpha power and binned into quintiles. Mean prestimulus alpha power and mean RT were calculated for each subject and each quintile, and then were averaged over subjects.

Again, debriefing after the experiment confirmed that participants were not able to detect whether the metallic cylinder used during intervention was the real magnet or the sham (43.3\% of correct guesses; Pearson $\chi^{2}$ test, $p=0.46$ ).

\section{Statistical analyses}

For the three experiments, alpha power or RT values exceeding 2.5 SDs from the group mean in each condition (baseline, intervention, or postintervention) were eliminated from further analyses $(1.8 \%, 3.6 \%$, and $2.4 \%$ of the alpha power data, respectively; and $2.3 \%, 2.9 \%$, and $2.5 \%$ of the RT data, respectively). To avoid statistical power problems, a multiple imputation method was used to treat some missing values before computing the ANOVA. Data normality was assessed by using a Shapiro-Wilk test. Due to the low numbers of errors $(<3 \%)$ expressed by all subjects in Experiments 2 and 3, errors rate were not analyzed further. 
In Experiment 1, the log-transformed relative alpha power-IAF $\pm 2 \mathrm{~Hz}$-was entered into a mixed ANOVA, separately for intervention and postintervention, with the betweensubjects factor "group" (real vs sham), and the within-subjects factors "region" (occipital and parietal), "electrode" (left, $\mathrm{O} 1$ and $\mathrm{P} 3$; right, $\mathrm{O}_{2}$ and $\mathrm{P} 4$; central, POZ and $\mathrm{Pz}$ ), and "time of stimulation" (arbitrary time bins T1, T2, T3, and T4). Note that the factor group was conservatively considered between subjects, despite the crossover design, to maintain the same statistical power between Experiment 1 and Experiment 3. Only occipital and parietal sites were included in this primary analysis due to the occipital localization of the magnet $[\mathrm{POz}$ (occipital cortex close to the parietal cortex)] and the occipitoparietal origin of alpha oscillations modulated by visual inputs. Nonetheless, to test the spatial specificity of tSMSdependent effects, the same ANOVAs were repeated with region (frontal and central) as a within-subject factor and with all other factors being equal. We also confirmed the alpha specificity of tSMS-dependent effects by performing the same ANOVA on delta $(2-4 \mathrm{~Hz})$, theta (4-6 Hz), alpha $(7-14 \mathrm{~Hz})$, and beta (15-30 $\mathrm{Hz}$ ) bands. Note that, because of the impossibility of identifying individual peaks for all bands and all subjects, fixed-frequency bands were used for this analysis (we also conservatively reanalyzed the alpha band).

In Experiment 2, the median RTs (for correct trials only) were calculated for each condition (intervention and postintervention) in each subject and then entered into a repeatedmeasures mixed ANOVA. Group (real vs sham) was the between-subjects factor; and the presence of the target (present vs absent), the number of the stimuli in the display or set size (low vs high), and the type of distractor ratio $(0: 48,6: 42,24: 24,42: 6$, and 48:0) were withinsubject factors. Significant interaction effects involving the group factor were further analyzed with post hoc $t$ test comparisons with Bonferroni correction and additional ANOVAs as appropriate.

In Experiment 3, alpha power analysis was conducted in the same way as in Experiment 1, including an additional analysis for the pause period. Similarly, behavioral analysis was performed as described for Experiment 2. In all cases, we used the same number of factors and levels. Additionally, the relationship between prestimulus alpha power and behavioral responses for both groups (real and sham) was further investigated using the single-trial analysis described in the data analysis section. All RT trials were separated into "easy task conditions" and "difficult task conditions" based on the distractor ratios showing significant differences between real and sham groups. The prestimulus alpha power and RT for easy and difficult task conditions were calculated for each subject in each quintile and then averaged over subjects. RTs were entered into a twoway mixed ANOVA, separately for easy and difficult task conditions, with quintile (within subjects) and group (between subjects) as factors.

Experiment 4 (control experiments and results)

Subjects and procedure. Effects of SMS on median nerve sensory action potential (SAP) recordings. We performed two separate control experi-

B
Alpha EEG Power

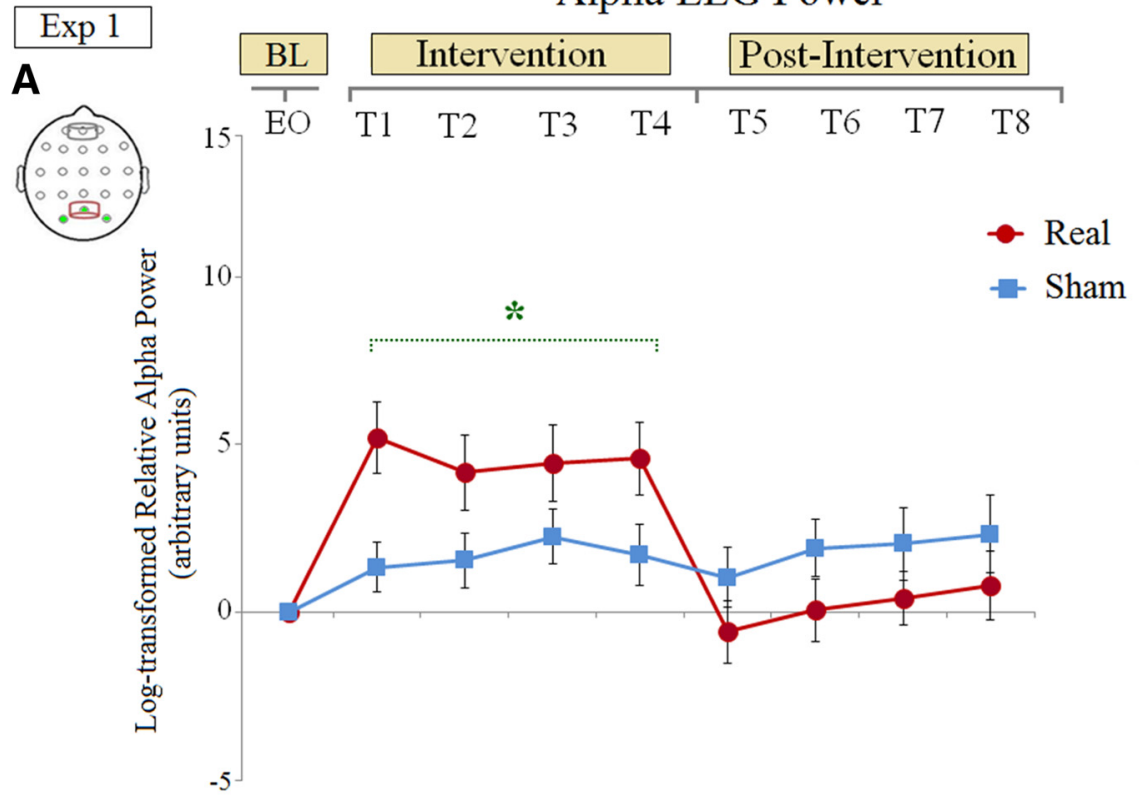

Individual Alpha Frequency (IAF)

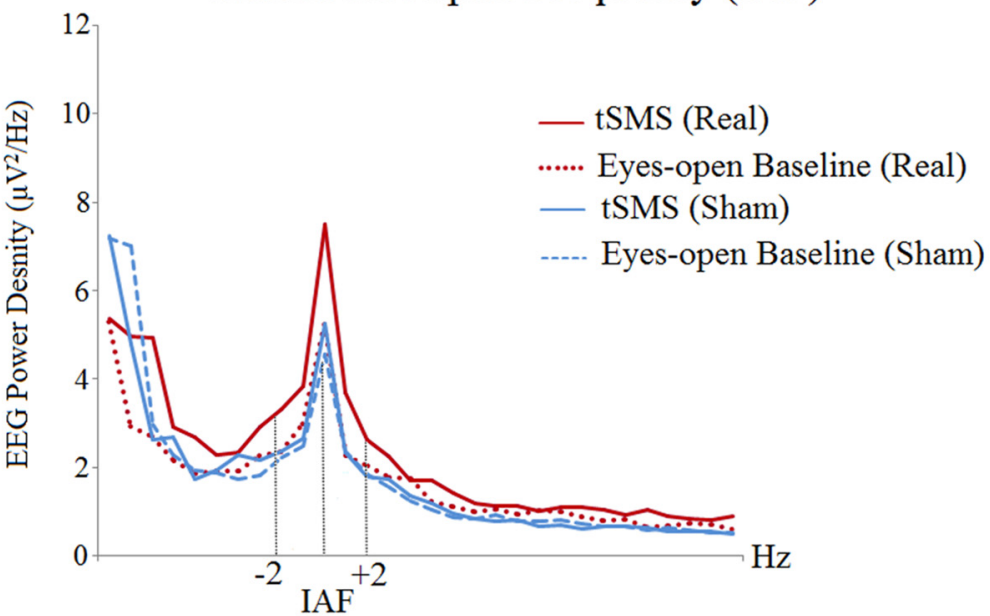

Figure 3. $\quad$ tSMS over the human visual cortex increases EEG alpha activity (Experiment 1). $\boldsymbol{A}$, Real tSMS (red) over visual cortex during the 10 min intervention session induced a relative increase in alpha EEG power compared with sham (blue) stimulation in wakeful rest. Plotted relative alpha power pertains to the group average of the three occipital electrodes showing statically significant differences between groups after post hoc analysis and Bonferroni correction. These are highlighted in green on electrode scalp maps (top left) with the positions of the magnet and frontal counterweight also indicated. The $p$ values $\left(^{*}\right)$ indicate the post hoc significance levels for the respective statistical comparisons region $\times$ group (occipital region real vs sham: $p<0.01$; see Table 1). Error bars here, and in all subsequent figures represent the SEM. EO, Eyes-open condition; BL, baseline. $\boldsymbol{B}$, Group-averaged EEG activity estimated from peak IAF. The plot depicts the grand average of the transformed EEG spectral power density values at occipital sites (01, 02, and P0z) for 2 min preceding (dotted lines) and during (solid lines) the intervention condition separately for the real (red) or sham (blue) tSMS groups. Real, but not sham, tSMS increased EEG alpha power (with alpha band defined here as $\mathrm{IAF} \pm 2 \mathrm{~Hz}$ ) in visual areas. Exp, Experiment.

ments to exclude the possibility that SMS could interfere with the EEG recording system. Our first experiment (Experiment 4.1) was designed to investigate the possible effects of SMS on the SAP amplitude (we used a standard methodology to obtain median nerve SAP). A second experiment (Experiment 4.2) was designed to assess possible effects of SMS on artificially induced $8 \mathrm{~Hz}$ oscillations of the SAPs (we used a modified methodology to obtain median nerve SAPs at a frequency of $8 \mathrm{~Hz}$ ). For both experiments (Experiments 4.1 and 4.2), the median nerve stimulation was performed using ring electrodes with conductive gel. The cathode was positioned proximally on the distal region of the middle phalanx of the third finger, and the anode on the distal phalanx of the same finger $\sim 2 \mathrm{~cm}$ from the negative electrode. The stimulus consisted of $0.2 \mathrm{~ms}$ 
A

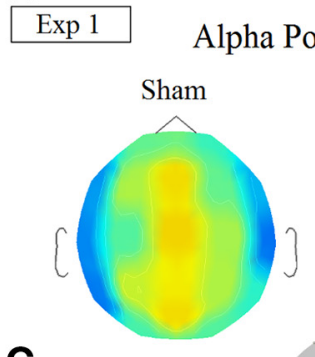

C

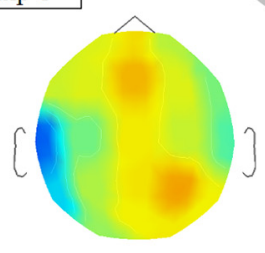

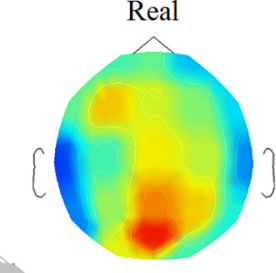

.

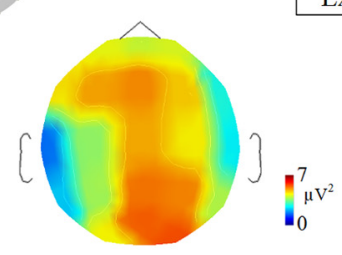

B

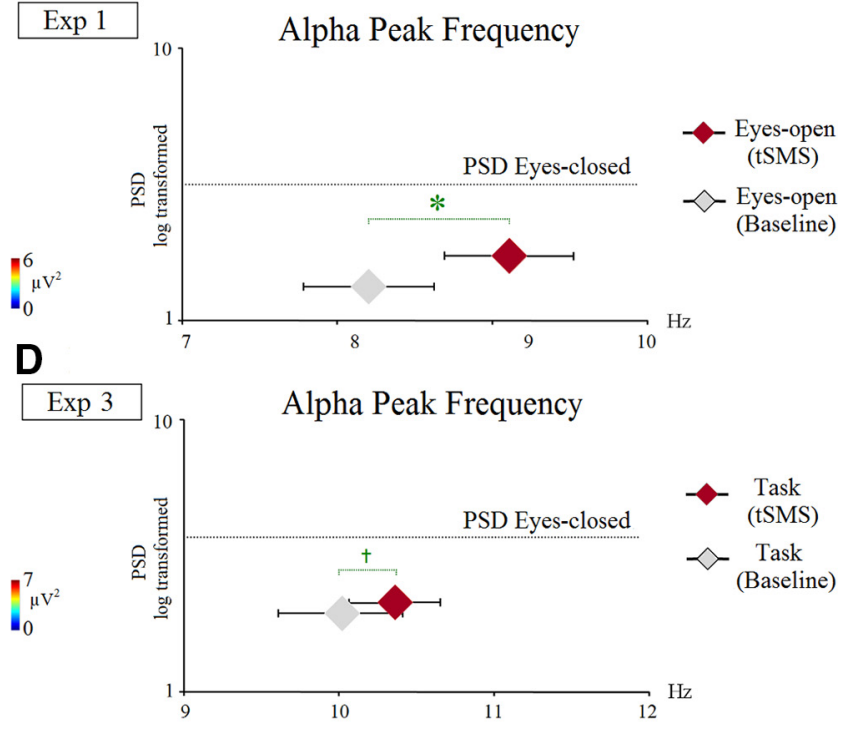

Figure 4. Alpha power density and alpha peak frequencies. $A, C$, Scalp topography of average EEG alpha power in sham and real groups during the tSMS intervention session in Experiment 1 (10 min eyes-open resting EEG) and Experiment 3 (performing the 10 min visual search task). Real relative to sham tSMS delivery over the visual cortex produced a focal, significant increase in scalp EEG alpha power at occipital sites in Experiment $1(\boldsymbol{A})$, whereas alpha power was significantly higher over parietal-occipital sites in Experiment 3 ( $\boldsymbol{C}$. Because of the difference in the average amplitude of alpha power density during Experiment 1 (eyes-open resting EEG) and Experiment 3 (attentional task performance), and to maximize the visualization of the effects between real and sham conditions, the topographies of these two experiments were plotted on different scales. $\boldsymbol{B}$, Peak frequency of the alpha rhythm in Experiment 1 was shifted from 8.2 Hz during eyes-open baseline to $9.1 \mathrm{~Hz}$ during real tSMS, which approaches the alpha peak of the eyes-closed baseline value $(10.2 \mathrm{~Hz})$. D , The same effect was observed in Experiment 3 comparing preintervention (task baseline) and intervention (tSMS) periods during the performance of the visual search task, although this did not reach significance. Exp, Experiment; PSD, power spectrum density. * $p=0.03$; $†$ statistical trend.

rectangular pulses. The intensity was chosen to obtain slightly submaximal SAP amplitude (to optimize the possibility to see amplitude changes). Recordings were obtained from 10 median nerves of five normal volunteers (four women; mean \pm SD age, $36.4 \pm 7.4$ years; age range, 29-44 years) for Experiment 4.1, and from 8 median nerves obtained from five normal volunteers (five women; mean age, $35.6 \pm 6.2$ years; age range, 29-44 years) for Experiment 4.2.

Experiment 4.1. A schematic of the experimental setup is shown in Figure $2 A$. SAP recordings were made using silver chloride cup electrodes located over the median nerve at the wrist. The stimulation frequency was $0.4 \mathrm{~Hz}$, and stimuli were delivered for $300 \mathrm{~s}$ at baseline followed by two periods of $300 \mathrm{~s}$ during SMS. A total of 120 SAPs were obtained and averaged for baseline ( $5 \mathrm{~min}$ ), and two blocks of 120 SAPs were obtained during the SMS (10 min). During the baseline period, the sham metallic cylinder was placed over the recording electrodes using a customized cardboard box. During SMS, the sham metallic cylinder was substituted with the rMag60. SAP amplitudes were virtually identical at baseline and during SMS (means are reported in Fig. $2 B$, and a representative subject is displayed in Fig. 2C).

Experiment 4.2. A schematic of the experimental setup is shown in Figure $2 D$. SAP recordings were made using two of the same EEG cap electrodes used for the EEG experiments. The two electrodes were located over the median nerve at the wrist. The stimulation protocol was a train of 40 stimuli at $8 \mathrm{~Hz}(5 \mathrm{~s})$ followed by $30 \mathrm{~s}$ of rest (Fig. $2 E)$. This protocol was repeated 17 times for baseline $(10 \mathrm{~min})$ and 17 times during the SMS (10 $\mathrm{min})$. A total of 680 SAPs were obtained and averaged for baseline (10 $\mathrm{min})$, and 680 SAPs were obtained during the SMS (10 $\mathrm{min}$ ). During baseline, the sham metallic cylinder was placed over the recording electrodes as in Experiment 4.1. During the SMS, the sham metallic cylinder was substituted with the Mag60r. Again, SAP amplitudes evoked during baseline and during SMS were identical (Fig. 2F). Moreover, after stimulus artifact removal, we obtained a power spectrum from the 17 stimulation periods both at baseline and during SMS. Power spectra showed the artificially induced $8 \mathrm{~Hz}$ oscillations, and they were identical at baseline and during SMS (Fig. 2G). With these control experiments, we exclude the possibility that tSMS had any effects on the recording systems.

\section{Table 1. EEG results}

\begin{tabular}{lll}
\hline & Intervention & Postintervention \\
\hline $\begin{array}{ll}\text { Experiment } 1 \\
\text { Group }\end{array}$ & $F_{(1,28)}=2.2, p=0.14$ & $F_{(1,28)}=2.6, p=0.11$ \\
Region $\times$ group & $F_{(1,28)}=6.7, p=0.01^{*}$ & $F_{(1,28)}=1.0, p=0.32$ \\
$\quad$ Electrode $\times$ group & $F_{(2,56)}=0.1, p=0.82$ & $F_{(2,56)}=0.7, p=0.61$ \\
Time $\times$ group & $F_{(3,84)}=1.5, p=0.25$ & $F_{(3,84)}=0.2, p=0.85$ \\
Region $\times$ electrode $\times$ group & $F_{(2,56)}=0.8, p=0.45$ & $F_{(2,56)}=0.6, p=0.69$ \\
Region $\times$ time $\times$ group & $F_{(3,84)}=0.2, p=0.90$ & $F_{(3,84)}=0.2, p=0.85$ \\
Electrode $\times$ time $\times$ group & $F_{(6,168)}=0.8, p=0.54$ & $F_{(6,168)}=1.3, p=0.32$ \\
Region $\times$ electrode $\times$ time & $F_{(6,108)}=1.2, p=0.30$ & $F_{(6,108)}=0.7, p=0.60$ \\
$\quad \times$ group & & \\
Experiment 3 & & \\
Group & $F_{(1,27)}=0.7, p=0.42$ & $F_{(1,27)}=0.1, p=0.9$ \\
Region $\times$ group & $F_{(1,27)}=1.3, p=0.27$ & $F_{(1,27)}=0.2, p=0.67$ \\
Electrode $\times$ group & $F_{(2,54)}=4.7, p=0.01 *$ & $F_{(2,54)}=2.1, p=0.14$ \\
Time $\times$ group & $F_{(3,81)}=3.6, p=0.02 *$ & $F_{(3,81)}=0.5, p=0.68$ \\
Region $\times$ electrode $\times$ group & $F_{(2,54)}=0.7, p=0.48$ & $F_{(2,54)}=1.9, p=0.17$ \\
Region $\times$ time $\times$ group & $F_{(6,164)}=0.4, p=0.69$ & $F_{(6,162)}=2.4, p=0.06 \dagger$ \\
$\quad$ Electrode $\times$ time $\times$ group & $F_{(6,162)}=1.9, p=0.13$ & $F_{(3,81)}=3.4, p=0.04$ \\
Region $\times$ electrode $\times$ time & $F_{(6,162)}=0.5, p=0.68$ & $F_{(6,162)}=1.5, p=0.22$ \\
$\quad \times$ group & & \\
\hline
\end{tabular}

ANOVA effects involving group factor in Experiments 1 and 3 during intervention and postintervention periods within the frequency range of the IAF and from occipitoparietal regions. Bold numbers indicate significant ANOVA effects. $p$ values are shown after Greenhouse-Geisser correction.

*Highest-order interaction effect for significance that was used to explain our results after post hoc analysis. †A statistical trend.

\section{Results}

Experiment 1-modulation of EEG alpha power by tSMS applied to the visual cortex

In Experiment 1, participants underwent either tSMS or sham tSMS on separate days in a within-subjects design (Fig. 1A). We recorded EEG activity in a $4 \mathrm{~min}$ baseline ( 2 min eyes closed and 


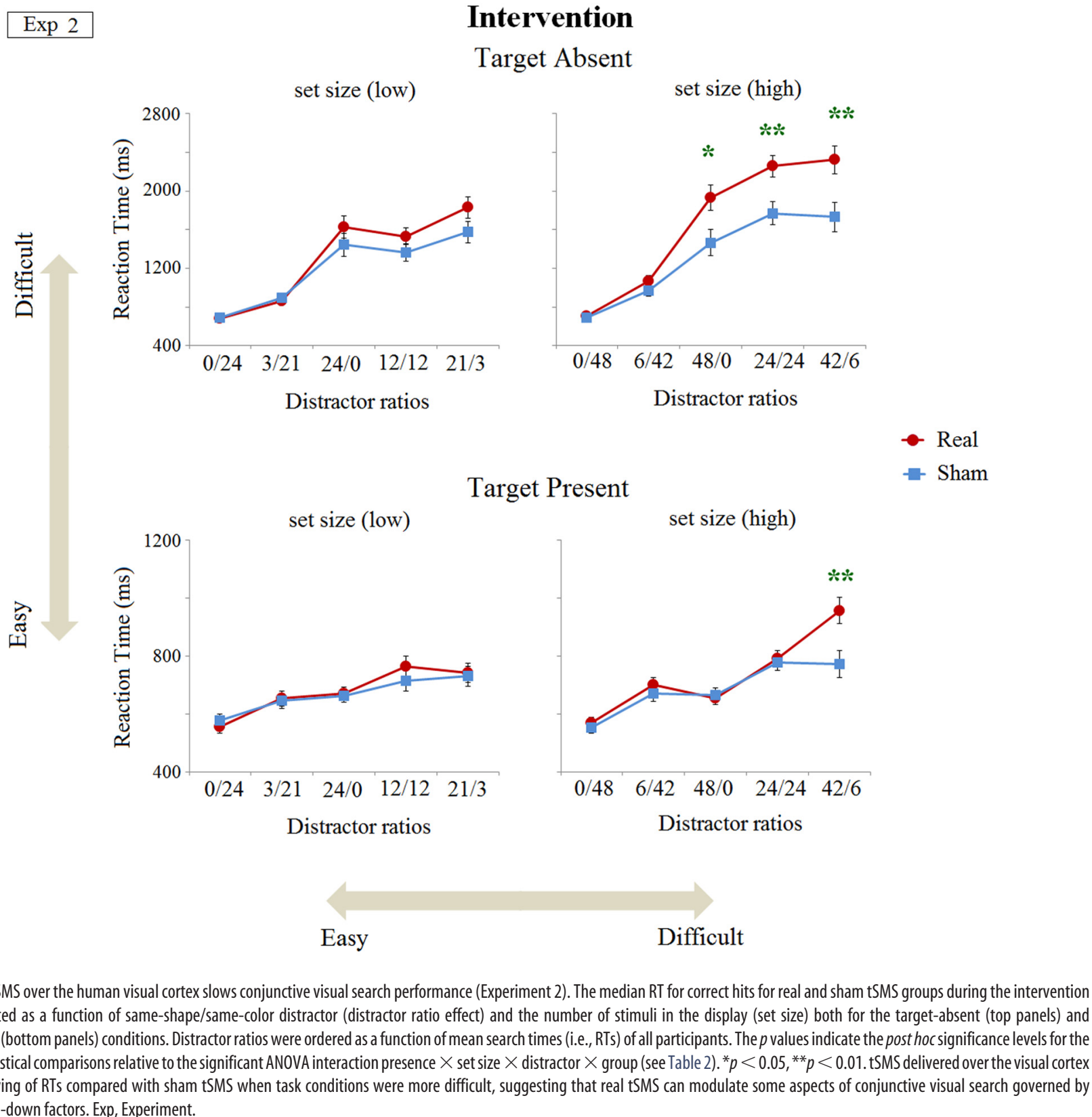

Figure 5. TSMS over the human visual cortex slows conjunctive visual search performance (Experiment 2). The median RT for correct hits for real and sham tSMS groups during the intervention session is plotted as a function of same-shape/same-color distractor (distractor ratio effect) and the number of stimuli in the display (set size) both for the target-absent (top panels) and target-present (bottom panels) conditions. Distractor ratios were ordered as a function of mean search times (i.e., RTs) of all participants. The $p$ values indicate the post hoc significance levels for the respective statistical comparisons relative to the significant ANOVA interaction presence $\times$ set size $\times$ distractor $\times$ group (see Table 2$) .{ }^{*} p<0.05$, ${ }^{* *} p<0.01$. tSMS delivered over the visual cortex induced a slowing of RTs compared with sham tSMS when task conditions were more difficult, suggesting that real tSMS can modulate some aspects of conjunctive visual search governed by attentional top-down factors. Exp, Experiment.

2 min eyes open), followed by a 10 min intervention (real or sham) and by a 10 min postintervention period. Analyses were restricted to parietal and occipital electrodes, focusing on alphaband activity (IAF, $\pm 2 \mathrm{~Hz}$, log-transformed and normalized to eyes-open baseline values).

Strikingly, tSMS increased alpha EEG activity relative to sham tSMS during the intervention (Fig. $3 A, B$ ). This effect was spatially selective (interaction region $\times$ group: $F_{(1,28)}=6.7, p=$ $0.01)$, being observed under tSMS over occipital $(p<0.01)$ but not parietal ( $p=0.73$ ) electrodes (Fig. $4 A$, Table 1$)$. Alpha enhancement was evident in all four arbitrarily defined 2.5 min time windows (T1-T4) during the intervention session (Fig. 3A). Note that the tSMS-induced alpha power increase is a fraction of the massive alpha increase induced by eye closing (Niedermeyer, 1993; Wang, 2010; see Fig. 4B). Postintervention, alpha power immediately returned to values close to eyes-open baseline values (Fig. 3A). In addition, we observed that tSMS significantly increased not only the alpha power, but also the peak frequency of the alpha rhythm, which shifted from (mean \pm SD) $8.2 \pm 1.6 \mathrm{~Hz}$ during the eyes-open baseline period to $9.1 \pm 1.6 \mathrm{~Hz}$ during the tSMS (paired $t$ test, $p=0.03$; Fig. $4 B$ ). The increase in alpha peak frequency is unlikely to be due to artifactual interference between tSMS and the EEG recording system. Any possible artifactual origin of the observed increase in alpha power was previously excluded in a separate control study (see Experiment 4 in Materials and Methods; Fig. 2). To further test the spatial specificity of the increase in EEG alpha power during tSMS, we repeated the same analysis on central and frontal electrodes, obtaining no significant differences between real and sham groups (group: $F_{(1,28)}=$ $0.11 ; p=0.74$; region $\times$ group: $\left.F_{(1,28)}=0.27 ; p=0.61\right)$. We also confirmed the frequency specificity of the tSMS-dependent power increase by repeating the same analysis for delta $(2-4 \mathrm{~Hz})$, theta $(4-6 \mathrm{~Hz})$, alpha $(7-14 \mathrm{~Hz})$, and beta $(15-30 \mathrm{~Hz})$ bands (fixed-frequency bands). tSMS had no significant effect on delta (group: $\left.F_{(1,28)}=0.8 ; p=0.36\right)$, theta (group: $F_{(1,28)}=1.1 ; p=$ 0.30 ), or beta (group: $F_{(1,28)}=0.1 ; p=0.85$ ) oscillations. How- 
ever, in this analysis using fixed-frequency bands, we confirmed the tSMS-dependent power increase of alpha oscillations (group: $\left.F_{(1,28)}=5.4 ; p=0.02\right)$. We therefore show that the focal application of a static magnetic field over the visual cortex produces a spatially specific and frequency-specific increase in alpha power.

\section{Experiment 2-modulation of behavioral performance by tSMS applied to the visual cortex during visual search}

We next sought to characterize a functional correlate of the observed tSMS-dependent modulation of posterior alpha power. Participants were randomized to two groups (real and sham), adopting a between-subjects design (Fig. $1 B$ and Materials and Methods). Participants completed a conjunction visual search task with a distractor-ratio manipulation (Wolfe et al., 1989; Poisson and Wilkinson, 1992), a perceptual task engaging visual selective attention (Bacon and Egeth, 1997; Müller and Krummenacher, 2006) in which participants were required to identify a target (an orange square) among two classes of distractors (orange triangles and/or blue squares). That is, the target was defined by a particular combination of two highly discriminable features (color and form), and shared one of these features with each of the distractors. To vary the degree of cognitive load, the following three parameters were randomly manipulated across trials (Fig. 1D): (1) the presence or absence of target (target presence); (2) the fixed total number of stimuli that were presented in the display (set size); and (3) the relative frequency of the two types of distractors (distractor ratio). Participants were required to locate the target stimulus as quickly as possible and to indicate target presence or absence via a two-alternative button press. The task was performed preintervention, during the intervention (real or sham), and postintervention. We measured both accuracy and RTs.

Across both groups (real or sham), we observed highly accurate target detection over all trial types $(99.1 \pm 1.0 \%$ correct responses). As predicted, both groups were slower (longer RTs) for target-absent relative to present trials, and for more attentiondemanding trials (high set size and more difficult distractor ratios; Fig. 5). Preintervention, RTs did not differ between the real and sham groups. However, during the intervention, real tSMS induced a significant slowing of RTs compared with sham tSMS (Table 2), specifically for the most difficult task conditions (Fig. 5), as follows: trials with target present, high set size, and $42: 6$ distractor ratio $(p<0.01)$, as well as trials with target absent, high set size, and 48:0 $(p=0.02), 24: 24(p<0.01)$, and 42:6 $(p<$ 0.01 ) distractor ratios. Aftereffects of tSMS in behavior were present despite no change in alpha power postintervention, but these were weak, as suggested by RTs remaining generally slower for trials with high set size in the real group compared with sham $(p=0.03$; Table 2, Fig. $6 A)$. Thus, a focal static magnetic field over the visual cortex significantly slowed RTs in a visual conjunction search task only for the most attention-demanding trials.

\section{Experiment 3-combined modulation of alpha power and behavioral performance by tSMS}

Our motivation for testing tSMS effects on visual target detection followed our observation that this intervention increases occipital alpha power (Experiment 1) and previous observations (Ergenoglu et al., 2004; Hanslmayr et al., 2007) that prestimulus alpha power correlates inversely with subsequent target detection. Thus, having confirmed the behavioral effect of occipital tSMS on target detection (Experiment 2), we next examined the relationship between the tSMS-dependent increase in RT and the

\section{Table 2. Behavioral results}

\begin{tabular}{lcc}
\hline & Intervention & Postintervention \\
\hline Experiment 2 & & \\
$\quad$ Group & $F_{(1,27)}=4.8, p=\mathbf{0 . 0 4}$ & $F_{(1,27)}=3.9, p=0.06 \dagger$ \\
Presence $\times$ group & $F_{(1,27)}=5.3, p=\mathbf{0 . 0 3}$ & $F_{(1,27)}=2.7, p=0.11$ \\
Set size $\times$ group & $F_{(1,27)}=25.6, p<\mathbf{0 . 0 1}$ & $F_{(1,27)}=6.8, p=\mathbf{0 . 0 1}$ \\
Distractor $\times$ group & $F_{(4,108)}=4.4, p=\mathbf{0 . 0 3}$ & $F_{(4,108)}=2.1, p=0.12$ \\
Presence $\times$ set size $\times$ group & $F_{(1,27)}=10.2, p=\mathbf{0 . 0 3}$ & $F_{(1,27)}=1.0, p=0.33$ \\
Presence $\times$ distractor $\times$ group & $F_{(4,108)}=3.4, p=\mathbf{0 . 0 1}$ & $F_{(4,108)}=2.3, p=0.09$ \\
Set size $\times$ distractor $\times$ group & $F_{(4,108)}=2.7, p=\mathbf{0 . 0 5}$ & $F_{(4,108)}=0.5, p=0.58$ \\
Presence $\times$ set size $\times$ distractor & $F_{(4,108)}=2.7, p=\mathbf{0 . 0 3} *$ & $F_{(4,108)}=0.6, p=0.53$ \\
$\quad \times$ group & & \\
Experiment 3 & & \\
Group & & \\
Presence $\times$ group & $F_{(1,28)}=6.8, p=\mathbf{0 . 0 1}$ & $F_{(1,27)}=0.5, p=0.47$ \\
Set size $\times$ group & $F_{(1,28)}=5.8, p=\mathbf{0 . 0 2}$ & $F_{(1,27)}=0.6, p=0.42$ \\
Distractor $\times$ group & $F_{(1,28)}=6.5, p=\mathbf{0 . 0 2}$ & $F_{(1,27)}=3.9, p=0.06 \dagger$ \\
Presence $\times$ set size $\times$ group & $F_{(4,112)}=6.7, p=\mathbf{0 . 0 2}$ & $F_{(4,108)}=1.0, p=0.38$ \\
Presence $\times$ distractor $\times$ group & $F_{(1,28)}=3.4, p=0.07 \dagger$ & $F_{(1,27)}=6.7, p=0.02$ \\
Set size $\times$ distractor $\times$ group & $F_{(4,112)}=3.3, p=0.01$ & $F_{(4,108)}=1.4, p=0.25$ \\
Presence $\times$ set size $\times$ distractor & $F_{(4,112)}=2.8, p=\mathbf{0 . 0 3 *}$ & $F_{(4,108)}=2.3, p=0.08 \dagger$ \\
$\quad \times$ group & &
\end{tabular}

ANOVA effects involving group factor in Experiments 2 and 3 during intervention and postintervention periods. Bold numbers indicate significant ANOVA effects. $p$ values are shown after Greenhouse-Geisser correction.

*Highest-order significant effect that was used to guide post hoc analyses.

†A statistical trend.

tSMS-dependent increase in prestimulus alpha power, on a single-trial basis. Therefore, in Experiment 3 we repeated the visual search task while simultaneously recording EEG (Fig. 1C). The experimental paradigm was virtually identical to the combination of Experiments 1 and 2, except that the design was double blind (both subjects and experimenter were blind to the intervention) instead of single blind (only subjects were blind to the intervention), as in Experiments 1 and 2. Two brief pauses were also introduced during task performance, during which eyes-open resting EEG activity was recorded (Fig. $1 C$ ), leading to a longer intervention (12 min).

The results of Experiment 3 replicate and extend the main results obtained separately in Experiments 1 and 2. First, the main electrophysiological effect of tSMS delivered over the visual cortex was replicated, confirming the increase in alpha activity compared with sham tSMS during the intervention (Fig. $7 A, B$ ), with slightly later onset and a more right-lateralized effect compared with Experiment 1 (Fig. 4C, Table 1). These latter differences between experiments likely reflect the different experimental condition (task vs rest). Note that the differences between real and sham tSMS during the 2 min rest pauses between tasks did not reach statistical significance (Fig. 7A), likely reflecting a different resting condition compared with Experiment 1. The spatial specificity of the increase in EEG alpha power during tSMS was confirmed by the absence of significant differences between real and sham groups at central and frontal electrodes (group: $F_{(1,27)}=0.3 ; p=0.61$ ), $p=$ 0.31 ; region $\times$ group: $\left.F_{(1,27)}=0.2 ; p=0.66\right)$. The frequency specificity was also confirmed by the absence of tSMS-dependent differences for delta and beta oscillations, respectively (group: $F_{(1,27)}=$ $1.6 ; p=0.22$; and group: $F_{(1,27)}=0.2 ; p=0.67$ ). A trend for increased theta oscillations was observed during real tSMS compared with sham tSMS (electrode $\times$ group: $F_{(1,27)}=3.7 ; p=0.07$ ), likely due to the left tail of alpha-band activity, which again showed a tSMS-dependent power increase (region $\times$ electrode $\times$ group: $\left.F_{(2,54)}=5.4 ; p>0.01\right)$.

Second, the behavioral effect of tSMS was also replicated, with slower RTs compared with sham tSMS specifically for the most 
A $\operatorname{Exp}_{2}$

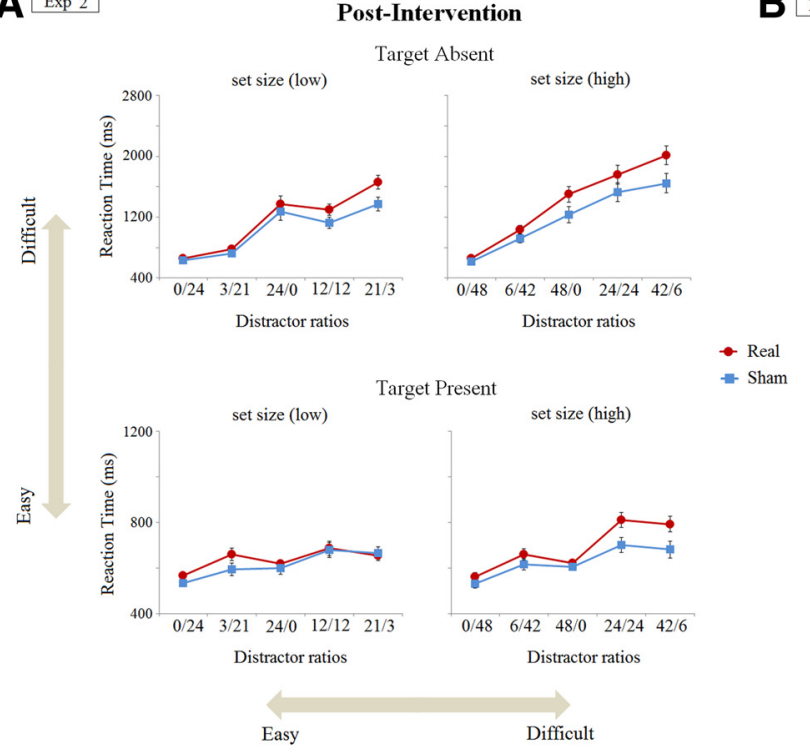

B $\operatorname{Exp}_{3}$
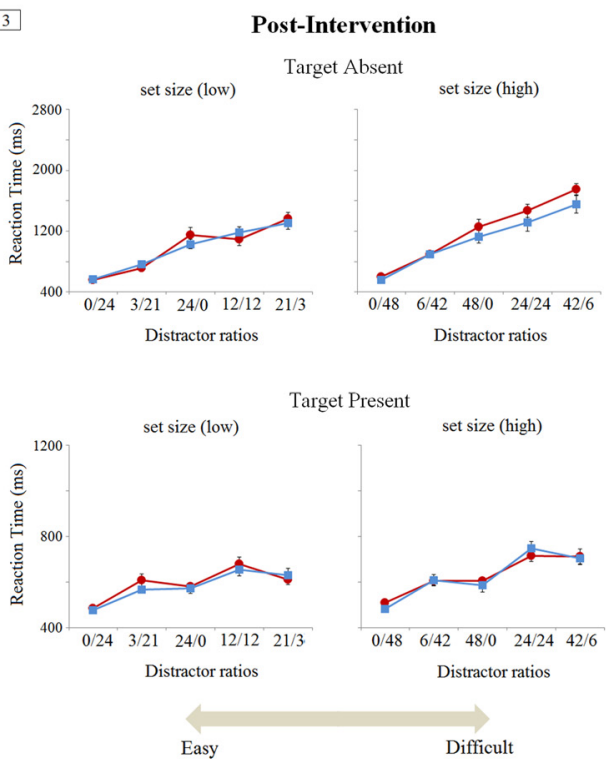

Figure 6. RTs during the postintervention. $\boldsymbol{A}, \boldsymbol{B}$, The median hit RT for real and sham tSMS groups during the postintervention period for Experiment $2(\boldsymbol{A})$ and Experiment $3(\boldsymbol{B})$. RTs are displayed as a function of same-shape/same-color distractor (distractor ratio effect), and the number of stimuli in the display (set size) both for the target-absent (top panels) and target-present (bottom panels) conditions. During the performance of the visual search task after the intervention period, most of the statistical differences between real and sham groups were no longer present, or only reached a statistical trend. In this respect, in Experiment 2, the ANOVA revealed a significant set size $\times$ group interaction $\left(F_{(1,27)}=6.8 ; p=0.01\right)$, with the real tSMS group taking longer to respond than the sham $\mathrm{SMMS}$ group for high set size trials $(p=0.03)$, but not for low set size trials $(p=0.12$; significant interaction is not indicated). In Experiment 3 , during the postintervention, there was a statistically significant presence $\times$ set size $\times$ group interaction $\left(F_{(1,27)}=6.7, p=0.02\right)$, but no post hoc test reached significance. Exp, Experiment.

difficult task conditions (Fig. 7C, Table 2): target-present, high set size, and 42: 6 distractor ratio $(p<0.01)$; and target absent, high set size, and 0: $48(p=0.03), 24: 24(p=0.03)$, and 42:6 distractor ratios $(p<0.01$; Fig. $7 C)$. No EEG and minimal behavioral effects were observed during the postintervention session (Fig. 6B, Table 2).

To investigate the relationship between tSMS-dependent modulation of alpha activity and behavioral performance, we next conducted a joint single-trial analysis of prestimulus alpha power and RT during the intervention session. In both groups, we confirmed the relationship between prestimulus posterior alpha-band power and RT described previously (Klimesch et al., 2007a; Snyder and Foxe, 2010; i.e., greater alpha power associated with slower response), particularly for more attention-demanding trials (difficult task conditions: quintile: $F_{(4,108)}=54.8: p<0.01$; Fig. $7 D)$. During tSMS, this relationship was shifted toward higher values of prestimulus alpha power, consequently leading to longer reaction times compared with sham tSMS in difficult task conditions (group: $F_{(1,27)}=5.1 ; p<0.01$ ), but not in easy task conditions (group: $F_{(1,27)}=0.3: p=0.64$; Fig. $7 D$ ). Even though additional mechanisms cannot be excluded, these data establish a correlative link between increased alpha power and decreased behavioral performance during tSMS.

\section{Discussion}

Overall, our data show that tSMS applied to the visual cortex increases alpha oscillations (Experiment 1) and induces behavioral effects in humans (Experiment 2). Importantly, we experimentally discarded the possibility that the increased alpha oscillations could be due to artifactual interference between tSMS and the EEG recording system (control experiments). The electrophysiological and behavioral effects of tSMS were replicated by simultaneous recording of visual search performance and EEG activity with a double-blind design (Experiment 3), demonstrating the robustness of our findings. These data suggest that the tSMS-dependent slowing of performance in a highly attention-demanding visual search is related to an increase of alpha oscillations.

Our first experiment demonstrated that tSMS interacts with the normal alpha oscillatory EEG activity of the occipital cortex during eyes-open rest. This interaction was fast (within minutes), lasted for the stimulation period, and disappeared postintervention, suggesting that tSMS can alter the relationship between a state of resting wakefulness and the presence of a dominant alpha rhythm in the EEG. Importantly, the effect was both spatially localized and frequency specific (i.e., we did not observe any tSMS-dependent power increases at delta, theta, or beta frequencies). Because increased occipital alpha activity reflects a state of reduced cortical excitability (Romei et al., 2008a,b), our results are in agreement with previous human studies demonstrating tSMS-induced reduction of motor cortex excitability (Oliviero et al., 2011; Silbert et al., 2013). During tSMS, alpha oscillations not only increased in amplitude, suggesting an increased number of neurons participating in the oscillations (Hindriks and van Putten, 2013), but also were right shifted in frequency, which could reflect a reduced temporal spacing of spikes underlying the oscillations (Hindriks and van Putten, 2013). At the cellular level, animal experiments have previously shown that static magnetic fields directly interfere with the functioning of membrane ion channels and consequently with the generation of action potentials (Rosen and Lubowsky, 1987; Coots et al., 2004; Yang et al., 2011). Therefore, tSMS could affect the delicate equilibrium between synchronous excitatory and inhibitory inputs at cortical pyramidal dendrites, thereby altering the large-scale spontaneous fluctuations of ongoing cortical (alpha) activity.

Our second experiment demonstrated that tSMS during a visual search task affects behavioral performance, specifically slowing the detection of a target stimulus among distractors. Critically, the slower performance (compared with sham) only 


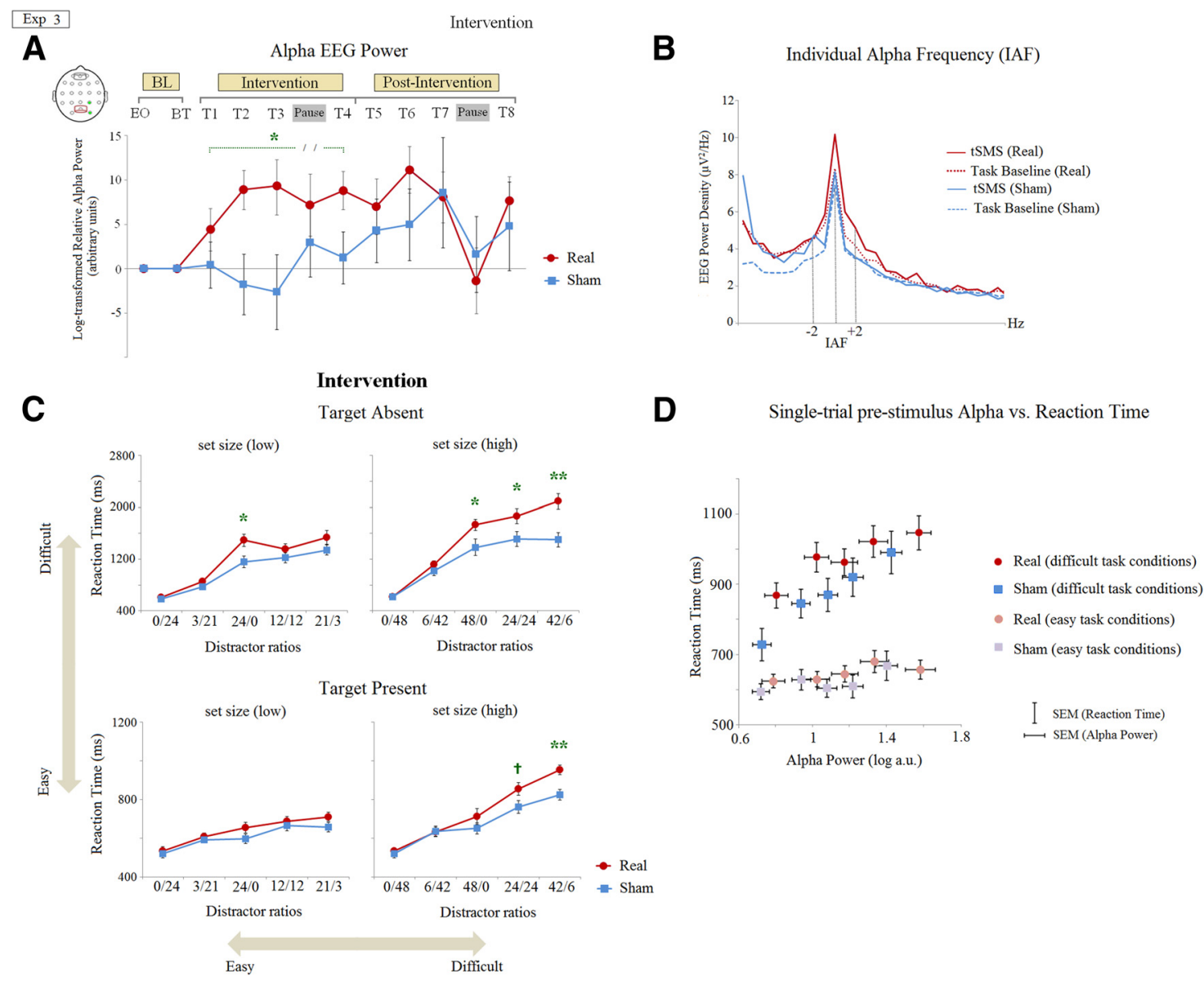

Figure 7. Prestimulus alpha oscillations predict conjunctive visual search performance (Experiment 3). $\boldsymbol{A}$, Real-tSMS (red) over visual cortex during the 12 min intervention session induced a relative increase in alpha EEG power compared with sham ISMS (blue) during visual search. Plot illustrates the enhancement in the relative alpha power for electrodes (highlighted in green on electrode scalp maps, as well as their position relative to the magnet) showing statically significant differences between groups after post hoc Bonferroni adjustment (electrode $\times$ group; right electrodes real vs sham: ${ }^{*} p=0.01$; see Table 1). $\boldsymbol{B}$, Group-averaged EEG activity estimated from individual peak alpha frequency. Real, but not sham, $\mathrm{tSMS}$ increased EEG alpha power (IAF $\pm 2 \mathrm{~Hz}$ ) in visual areas. Plots depict the grand average of the transformed EEG spectral power density values at occipital sites ( 02 and POz) for 2 min preceding (dotted lines) and during (solid lines) the intervention condition separately for the real (red) or sham (blue) tSMS groups. C, Effects of tSMS on visual search performance were replicated in Experiment 3: $t S M S$-induced slowing of visual search (mean RT for corrects hits) is observed when there are a higher number of same-shape and same-color distractors. Asterisks ${ }^{*}$ ) represent significant post hoc comparisons for the interaction presence $\times$ set size $\times$ distractor $\times$ group (see Table 2). D, Pooled single-trial data demonstrating a monotonic relationship between prestimulus alpha power and RT. For each subject, all correct hit trials during the intervention session were separately sorted according to prestimulus alpha power and were binned into quintiles. For each quintile, mean alpha power (collapsed over occipital electrodes) and mean RT were calculated. Conditions showing significant behavioral effects (difficult distractor ratios vs easy distractor ratios) between groups were collapsed, and separately calculated and plotted. Alpha power of the quintile scores are displayed in log scale. The typical relationship between alpha power and performance, observed during the difficult task conditions, was shifted toward higher values of prestimulus alpha power during tSMS, at least partly explaining the tSMS-induced increase in RTs. In all plots, $p$ values indicate the post hoc significance levels for the respective statistical comparisons. ${ }^{*} p<0.05,{ }^{* *} p<0.01$, ttrend). E0, Eyes-open condition; Exp, experiment; a.u., arbitrary units.

emerged on trials with the most difficult task conditions. Previous visual search studies have suggested that the detection of relatively easy targets relies upon bottom-up mechanisms, whereas the detection of more difficult targets requires top-down control (Nakayama and Silverman, 1986; Treisman and Sato, 1990; Shen et al., 2007) over the visual cortex from higher-order attention areas (Donner et al., 2002; Kalla et al., 2009; Wei et al., 2011). Our findings are compatible with a view that the decrease of cortical excitability induced by tSMS of the visual cortex does not affect the basic bottom-up processing of visual inputs, but it does interfere with the more sophisticated ability of higher-order attention areas to control the visual cortex.

In our third experiment, we directly linked the tSMS-induced EEG and behavioral changes by replicating the visual search task while simultaneously recording the EEG. A separate line of evidence suggests that top-down attentional processing during visual search and tasks requiring visuospatial attention involves the suppression of alpha activity in visual cortex (Thut et al., 2006;
Min and Herrmann, 2007; Capotosto et al., 2009; Roberts et al., 2014). Joint single-trial analysis confirmed the known relationship between alpha power and reaction time (i.e., the more the alpha power, the slower the response). Importantly, in easy task conditions the RTs were only slightly slower with higher alpha power, whereas in difficult task conditions the RTs were considerably slower with higher alpha power, likely due to the contribution of top-down processing (Min and Herrmann, 2007; Roberts et al., 2014). This explains why, even though tSMS increased the alpha power for all task conditions, it produced a significant slowing of reaction times only for the most difficult task conditions. Therefore, the selective tSMS effect on difficult — but not easy — target detection is consistent with the visual cortex becoming less sensitive to top-down control from higherorder attentional areas, at least partly due to the tSMS-induced increase of posterior alpha activity.

Based on these findings, we speculate that the most likely mechanism through which tSMS evokes the observed behavioral 
effects is a transient synchronization of corticosubcortical neural generators of alpha activity. Additional mechanisms can obviously not be excluded. In this sense, it is worth noting that the tSMS-induced effects on EEG alpha activity disappeared in the postintervention period, whereas the effects of tSMS on behavior lasted longer, in agreement with previous findings of tSMS on the excitability of the human sensorimotor cortex (Oliviero et al., 2011; Silbert et al., 2013; Kirimoto et al., 2014). Even though the full mechanisms of these alterations remain to be fully established, the present data show that tSMS affects both alpha EEG activity and human behavioral performance. In comparison with other noninvasive neuromodulation techniques, such as transcranial direct current stimulation or repetitive TMS (Paulus et al., 2013), tSMS induces these electrophysiological and behavioral effects without using any electrical current or time-varying magnetic pulses.

We conclude that focal static magnetic fields can interfere with normal brain function even at an intensity of 120-200 mT (at 2-3 cm from the magnet surface), offering a novel safe technique, which is both portable and inexpensive, to influence human brain activity and behavior.

\section{References}

Aguila J, Cudeiro J, Rivadulla C (2014) Effects of static magnetic fields on the visual cortex: reversible visual deficits and reduction of neuronal activity. Cereb Cortex. Advance online publication. Retrieved May 14, 2015. doi:10.1093/cercor/bhu228. CrossRef Medline

Bacon WJ, Egeth HE (1997) Goal-directed guidance of attention: evidence from conjunctive visual search. J Exp Psychol Hum Percept Perform 23: 948-961. CrossRef Medline

Barry RJ, Clarke AR, Johnstone SJ, Magee CA, Rushby JA (2007) EEG differences between eyes-closed and eyes-open resting conditions. Clin Neurophysiol 118:2765-2773. CrossRef Medline

Basar E (1980) EEG brain dynamics. Relation between EEG and brain evoked potentials. Amsterdam: Elsevier

Capotosto P, Babiloni C, Romani GL, Corbetta M (2009) Frontoparietal cortex controls spatial attention through modulation of anticipatory alpha rhythms. J Neurosci 29:5863-5872. CrossRef Medline

Coots A, Shi R, Rosen AD (2004) ofa0.5-T static magnetic field on conduction in guinea pig spinal cord. J Neurol Sci 222:55-57. CrossRef Medline

Donner TH, Kettermann A, Diesch E, Ostendorf F, Villringer A, Brandt SA (2002) Visual feature and conjunction searches of equal difficulty engage only partially overlapping frontoparietal networks. Neuroimage 15:16-25. CrossRef Medline

Dugué L, Marque P, VanRullen R (2011) The phase of ongoing oscillations mediates the causal relation between brain excitation and visual perception. J Neurosci 31:11889-11893. CrossRef Medline

Ergenoglu T, Demiralp T, Bayraktaroglu Z, Ergen M, Beydagi H, Uresin Y (2004) Alpha rhythm of the EEG modulates visual detection performance in humans. Brain Res Cogn Brain Res 20:376-383. CrossRef Medline

Foxe JJ, Snyder AC (2011) The role of alpha-band brain oscillations as a sensory suppression mechanism during elective attention. Front Psychol 2:154. CrossRef Medline

Foxe JJ, Simpson GV, Ahlfors SP (1998) Parieto-occipital approximately 10 $\mathrm{Hz}$ activity reflects anticipatory state of visual attention mechanisms. Neuroreport 9:3929-3933. CrossRef Medline

Häfeli U (2007) The history of magnetism in medicine. In: Magnetism in medicine: a handbook, Ed 2 (Andra W, Nowak H, eds), pp 3-25. Weiheim, Germany: Wiley-VCH Verlag GmbH \& Co. KGaA.

Hanslmayr S, Aslan A, Staudigl T, Klimesch W, Herrmann CS, Bäuml KH (2007) Prestimulus oscillations predict visual perception performance between and within subjects. Neuroimage 37:1465-1473. CrossRef Medline

Hindriks R, van Putten MJ (2013) Thalamo-cortical mechanisms underlying changes in amplitude and frequency of human alphao scillations. Neuroimage 70:150-163. CrossRef Medline

Kalla R, Muggleton NG, Cowey A, Walsh V (2009) Human dorsolateral prefrontal cortex is involved in visual search for conjunctions but not features: a theta TMS study. Cortex 45:1085-1090. CrossRef Medline

Kirimoto H, Tamaki H, Matsumoto T, Sugawara K, Suzuki M, Oyama M, Onishi H (2014) Effect of transcranial static magnetic field stimulation over the sensorimotor cortex on somatosensory evoked potentials in humans. Brain Stimul 7:836-840. CrossRef Medline

Klimesch W (1999) EEG alpha and theta oscillations reflect cognitive and memory performance: a review and analysis. Brain Res Brain Res Rev 29:169-195. CrossRef Medline

Klimesch W, Sauseng P, Hanslmayr S (2007a) EEG alpha oscillations: the inhibition-timing hypothesis. Brain Res Rev 53:63-88. CrossRef Medline

Klimesch W, Sauseng P, Hanslmayr S, Gruber W, Freunberger R (2007b) Event-related phase reorganization may explain evoked neural dynamics. Neurosci Biobehav Rev 31:1003-1016. CrossRef Medline

Makeig S, Bell AJ, Jung T-P, Sejnowski TJ (1996) Independent component analysis of electroencefalografic data. In: Advances in neural information processing systems, Vol 8 (Touretzky D, Mozer M, Hasselmo M, eds), pp 145-151. Cambridge, MA: MIT.

McLean MJ, Engström S, Holcomb RR, Sanchez D (2003) A static magnetic field modulates severity of audiogenic seizures and anticonvulsant effects of phenytoin in DBA/2 mice. Epilepsy Res 55:105-116. CrossRef Medline

McLean MJ, Engström S, Qinkun Z, Spankovich C, Polley D (2008) Effects of a static magnetic field on audiogenic seizures in black Swiss mice. Epilepsy Res 80:119-131. CrossRef Medline

Min BK, Herrmann CS (2007) Prestimulus EEG alpha activity reflects prestimulus top-down processing. Neurosci Lett 422:131-135. CrossRef Medline

Min BK, Busch NA, Debener S, Kranczioch C, Hanslmayr S, Engel AK, Herrmann CS (2007) The best of both worlds: phase-reset of human EEG alpha activity and additive power contribute to ERP. Int J Psychophysiol 65:58-68. CrossRef Medline

Müller HJ, Krummenacher J (2006) Visual search and selective attention. Vis Cogn 14:389-410. CrossRef

Nakayama K, Silverman GH (1986) Serial and parallel processing of visual feature conjunctions. Nature 320:264-265. CrossRef Medline

Niedermeyer E (1993) The normal EEG of the waking adult. In: Electroencephalography: basic principles, clinical applications, and related fields (Niedermeyer E, Lopes da Silva F, eds), pp 97-117. Baltimore: Williams and Wilkins.

Oliviero A, Mordillo-Mateos L, Arias P, Panyavin I, Foffani G, Aguilar J (2011) Transcranial static magnetic field stimulation of the human motor cortex. J Physiol 589:4949-4958. CrossRef Medline

Oliviero A, Carrasco-López MC, Campolo M, Perez-Borrego YA, Soto-León V, Gonzalez-Rosa JJ, Higuero AM, Strange BA, Abad-Rodriguez J, Foffani G (2014) Safety study of transcranial static magnetic field stimulation (tSMS) of the human cortex. Brain Stimul. Advance online publication. Retrieved May 14, 2015. doi:10.1016/j.brs.2014.12.002. CrossRef Medline

Paulus W, Peterchev AV, Ridding M (2013) Transcranial electric and magnetic stimulation: technique and paradigms. Handb Clin Neurol 116: 329-342. CrossRef Medline

Pfurtscheller G (2001) Functional brain imaging based on ERD/ERS. Vision Res 41:1257-1260. CrossRef Medline

Pfurtscheller G (2003) Induced oscillations in the alpha band: functional meaning. Epilepsia 44 [Suppl 12]:2-8. Medline

Poisson ME, Wilkinson F (1992) Distractor ratio and grouping processes in visual conjunction search. Perception 21:21-38. CrossRef Medline

Rivadulla C, Foffani G, Oliviero A (2014) Magnetic field strength and reproducibility of neodymium magnets useful for transcranial staticmagnetic field stimulation of the human cortex. Neuromodulation 17:438-441. CrossRef Medline

Roberts DC, Marcelli V, Gillen JS, Carey JP, Della Santina CC, Zee DS (2011) MRI magnetic field stimulates rotational sensors of the brain. Curr Biol 21:1635-1640. CrossRef Medline

Roberts DM, Fedota JR, Buzzell GA, Parasuraman R, McDonald CG (2014) Prestimulus oscillations in the alpha band of the EEG are modulated by the difficulty of feature discrimination and predict activation of a sensory discrimination process. J Cogn Neurosci 26:1615-1628. CrossRef Medline

Romei V, Brodbeck V, Michel C, Amedi A, Pascual-Leone A, Thut G (2008a) Spontaneous fluctuations in posterior alpha-band EEG activity reflect variability in excitability of human visual areas. Cereb Cortex 18:20102018. CrossRef Medline 
Romei V, Rihs T, Brodbeck V, Thut G (2008b) Resting electroencephalogram alpha-power over posterior sites indexes baseline visual cortex excitability. Neuroreport 19:203-208. CrossRef Medline

Rosen AD (2003) Mechanism of action of moderate-intensity static magnetic fields on biological systems. Cell Biochem Biophys 39:163-173. CrossRef Medline

Rosen AD (2010) Studies on the effect of static magnetic fields on biological systems. PIERS Online 6:133-136. CrossRef

Rosen AD, Lubowsky J (1987) Magnetic field influence on central nervous system function. Exp Neurol 95:679-687. CrossRef Medline

Rosen AD, Lubowsky J (1990) Modification of spontaneous unit discharge in the lateral geniculate body by a magnetic field. Exp Neurol 108:261265. CrossRef Medline

Shen J, Reingold EM, Pomplun M (2003) Guidance of eye movements during conjunctive visual search: the distractor-ratio effect. Can J Exp Psychol 57:76-96. CrossRef Medline

Shen J, Elahipanah A, Reingold EM (2007) Effects of context and instruction on the guidance of eye movements during a conjunctive visual search task. In: Eye movements: a window on mind and brain (van Gompel R, Fischer M, Murray W, Hill R, eds), pp 597-616. Oxford: Elsevier.

Silbert BI, Pevcic DD, Patterson HI, Windnagel KA, Thickbroom GW (2013) Inverse correlation between resting motor threshold and corticomotor excitability after static magnetic stimulation of human motor cortex. Brain Stimul 6:817-820. CrossRef Medline

Snyder AC, Foxe JJ (2010) Anticipatory attentional suppression of visual features indexed by oscillatory alpha-band power increases: a highdensity electrical mapping study. J Neurosci 30:4024-4032. CrossRef Medline
Thut G, Nietzel A, Brandt SA, Pascual-Leone A (2006) Alpha-band electroencephalographic activity over occipital cortex indexes visuospatial attention bias and predicts visual target detection. J Neurosci 26:9494-9502. CrossRef Medline

Treisman A, Sato S (1990) Conjunction search revisited. J Exp Psychol Hum Percept Perform 16:459-478. CrossRef Medline

van Dijk H, Schoffelen JM, Oostenveld R, Jensen O (2008) Prestimulus oscillatory activity in the alpha band predicts visual discrimination ability. J Neurosci 28:1816-1823. CrossRef Medline

VanRullen R, Macdonald JS (2012) Perceptual echoes at $10 \mathrm{~Hz}$ in the human brain. Curr Biol 22:995-999. CrossRef Medline

Wang XJ (2010) Neurophysiological and computational principles of cortical rhythms in cognition. Physiol Rev 90:1195-1268. CrossRef Medline

Wei P, Müller HJ, Pollmann S, Zhou X (2011) Neural correlates of binding features within- or cross-dimensions in visual conjunction search: an fMRI study. Neuroimage 57:235-241. CrossRef Medline

Wieraszko A (2000) Dantrolene modulates the influence of steady magnetic fields on hippocampal evoked potentials in vitro. Bioelectromagnetics 21:175-182. CrossRef Medline

Wolfe JM, Cave KR, Franzel SL (1989) Guided search: an alternative to the feature integration model for visual search. J Exp Psychol Hum Percept Perform 15:419-433. CrossRef Medline

Wu LQ, Dickman JD (2012) Neural correlates of a magnetic sense. Science 336:1054-1057. CrossRef Medline

Yang Y, Yan Y, Zou X, Zhang C, Zhang H, Xu Y, Wang X, Janos P, Yang Z, Gu H (2011) Static magnetic field modulates rhythmic activities of a cluster of large local interneurons in Drosophila antennal lobe. J Neurophysiol 106:2127-2135. CrossRef Medline 